\title{
Characteristics of the Dirac eigenvalue distribution in dense two-color QCD
}

\author{
Kenji Fukushima* \\ Yukawa Institute for Theoretical Physics, Kyoto University, Kyoto 606-8502, Japan \\ E-mail: fuku@yukawa.kyoto-u.ac.jp
}

\begin{abstract}
We exposit the eigenvalue distribution of the lattice Dirac operator in Quantum Chromodynamics with two colors (i.e. two-color QCD). We explicitly calculate all the eigenvalues in the presence of finite quark chemical potential $\mu$ for a given gauge configuration on the finite-volume lattice. We make use of the Banks-Casher relations to relate the eigenvalue spectral density to the physical observables. Assuming the strong coupling limit, we exhibit the numerical results for a random gauge configuration in two-color QCD implemented by the staggered fermion formalism and confirm that our results agree well with the known estimate quantitatively.
\end{abstract}

The XXVI International Symposium on Lattice Field Theory

July 14-19 2008

Williamsburg, Virginia, USA

\footnotetext{
*Speaker.
} 


\section{Introduction}

Quantum Chromodynamics with two colors (two-color QCD) instead of three is a sophisticated practice ground for theorists to extract worthwhile information out of dense quark matter. We immediately hit on several reasons why we can believe so: First of all, numerous works on dense two-color QCD have almost established a firm understanding on the ground state of twocolor QCD by the analytical approach as well as the Monte-Carlo simulation [1, 2, 3, 4, 5, 6]. Second, the notorious sign problem of the Dirac determinant at $\mu \neq 0$ (where $\mu$ is the quark chemical potential) is not so harmful as genuine QCD, which makes it viable to perform the Monte-Carlo integration [3, 7]. Third, dense two-color matter realizes a bosonic baryon system leading to the Bose-Einstein condensation of the color-singlet diquark [2, 8]. This two-color superfluid phase is reminiscent of the three-color superconducting phase [9], for they both break the $U_{B}(1)$ symmetry. Finally, enlarged flavor symmetry earned by the pseudo-real nature of the SU(2) group that is called Pauli-Gürsey symmetry constrains two-color QCD at $m_{q}=\mu=0$. The interplay between the chiral and diquark sectors simplifies owing to the symmetry, which enables us to construct an effective model for two-color QCD with less ambiguity.

We usually define the order parameter and concern its expectation value to examine the phase structure with varying the external parameters such as the temperature $T$, the quark chemical potential $\mu$, the quark mass $m_{q}$, and so on. In this article we will look into the eigenvalue distribution of the Dirac operator and characterize the state of matter by the distribution pattern. It is long known that the eigenvalue spectrum is informative in the vacuum and the random matrix theory is capable of determining the low-lying spectrum, which has recently been extended to the finite density study [5]. It is not only the low-lying spectrum but also the whole spectral density that we will deal with here. The Monte-Carlo simulation generates a set of gauge field configurations each of which has a substantial weight on the partition function. One configuration corresponds to one value for a certain operator (the order parameter for example of our interest). The more configurations we accumulate, the more accurately we can improve the expectation value of the order parameter. Here, we would remind that the well-known Banks-Casher relation [10] yields the chiral condensate given in terms of the eigenvalue spectral density at the origin (i.e. $m_{q} \rightarrow 0$ ). It follows in turn that the order parameter makes use of only tiny amount of the entire information available from the spectrum. Hence, we will unveil detailed information in a special case of dense and cold $(T=0)$ quark matter with two colors.

\section{Two-Color QCD at Strong Coupling}

In the limit of the strong coupling the gauge action does not enter the dynamics and the partition function is simply given by the fermionic part;

$$
Z=\left\langle(\operatorname{det} \mathscr{D})^{N_{f}}\right\rangle_{U} \equiv \int \prod_{n, \mu} \mathrm{d} U_{\mu}(n)(\operatorname{det} \mathscr{D})^{N_{f}} .
$$

Here $\mathscr{D}$ is the Dirac operator. Although the strong coupling limit is a drastic approximation which neglects the gauge dynamics completely, the Dirac determinant alone with random gluon fields can grasp rich contents of quark matter not only in the two-color case [1, 4, 8] but also in the general case [11, 12, 13]. 
In a box with volume $V=L^{4}$ (here we choose $L=6$ ), the operator $\mathscr{D}$ is a $\left(4 N_{c} V\right) \times\left(4 N_{c} V\right)$ matrix. We denote the eigenvalue of $\mathscr{D}$ by $\lambda_{i}$, that is, $\mathscr{D} v_{i}=\lambda_{i} v_{i}$, where $i$ runs from 1 to $4 N_{c} V$. Then the Dirac determinant is given by the product of all the eigenvalues. It is easy to prove that det $\mathscr{D}$ in the SU(2) gauge theory takes a real value even at finite density where $\mathscr{D}$ loses the $\gamma_{5}$-Hermiticity, i.e. $\gamma_{5} \mathscr{D}(\mu) \gamma_{5}=\mathscr{D}^{\dagger}(-\mu) \neq \mathscr{D}^{\dagger}(\mu)$. The standard argument follows;

$$
\operatorname{det} \mathscr{D}(\mu)=\operatorname{det}\left[\left(C \sigma_{2} \gamma_{5}\right)^{-1} \mathscr{D}(\mu)\left(C \sigma_{2} \gamma_{5}\right)\right]=\operatorname{det} \mathscr{D}^{*}(\mu)=[\operatorname{det} \mathscr{D}(\mu)]^{*} .
$$

Here, to derive the above, the necessary relations are $\gamma_{5} \gamma_{\mu} \gamma_{5}=-\gamma_{\mu}, C \gamma_{\mu} C^{-1}=-\gamma_{\mu}^{T}$, and $\sigma_{2} U \sigma_{2}=$ $U^{*}$ where the last relation corresponds to the pseudo-real nature of the $\mathrm{SU}(2)$ group.

From this argument we see that two-color $\operatorname{det} \mathscr{D}(\mu)$ is real but not necessarily positive. The simulation thus entails an even number of $N_{f}$ so that $(\operatorname{det} \mathscr{D})^{N_{f}}$ is positive definite. The two-color determinant, however, buries a nice property of respective eigenvalues under the product. We can prove that, if $\lambda_{i}=m_{q}+\mathrm{i} \lambda_{i}^{\prime}$ is an eigenvalue of the Dirac determinant in two-color QCD, there appear $m_{q}-\mathrm{i} \lambda_{i}^{\prime}, m_{q}+\mathrm{i} \lambda_{i}^{\prime *}$, and $m_{q}-\mathrm{i} \lambda_{i}^{\prime *}$ simultaneously in the eigenvalue spectrum [3, 7]. The proof may break down when $\mathrm{i} \lambda_{i}^{\prime}$ is a real number; the eigenvectors for $m_{q}+\mathrm{i} \lambda_{i}^{\prime}$ and $m_{q}-\mathrm{i} \lambda_{i}^{\prime *}$ could not be independent. According to Ref. [3] the staggered fermion is safe from such a possibility but the Wilson fermion has only a pair of $m_{q}+\mathrm{i} \lambda_{i}^{\prime}$ and $m_{q}-\mathrm{i} \lambda_{i}^{\prime}$ instead of a complex quartet in that case of real $i \lambda_{i}^{\prime}$. Then the single-flavor Wilson fermion suffers the sign problem once either of real $m_{q}+\mathrm{i} \lambda_{i}^{\prime}$ and $m_{q}-\mathrm{i} \lambda_{i}^{\prime}$ is negative.

\section{Banks-Casher Relations}

\subsection{Chiral Condensate}

It is widely known that the chiral condensate has a close connection to the Dirac eigenvalue distribution via the Banks-Casher relation [10]. In the explicit presence of the source for the chiral condensate (i.e. mass term), the Dirac operator could be decomposed into the form of $\mathscr{D}[m]=$ $m_{q} 1+\mathscr{D}[0]$ whose eigenvalue is denoted as $\lambda_{i}=m_{q}+\mathrm{i} \lambda_{i}^{\prime}$. The chiral condensate per flavor is given by the derivative of $Z$ with respect to $m_{q}$, which leads us to

$$
\frac{1}{N_{f}}\langle\bar{\psi} \psi\rangle=-\frac{1}{N_{f} V} \frac{\partial}{\partial m} \ln Z=-\frac{1}{V}\left\langle\left\langle\left\langle\sum_{i} \frac{1}{\lambda_{i}}\right\rangle\right\rangle=\left\langle\left\langle\oint \frac{\mathrm{d} \lambda}{2 \pi \mathrm{i}} \frac{\pi \rho_{\chi}(\lambda)}{\lambda}\right\rangle\right\rangle,\right.
$$

where $\rho_{\chi}(\lambda)$ is the eigenvalue spectral density which is to be expressed in the complex plane as

$$
\rho_{\chi}(\lambda) \equiv \frac{1}{\pi V} \sum_{i} \frac{1}{\lambda_{i}-\lambda},
$$

which is, strictly speaking, the resolvent rather than the spectral density. To keep the analogy to the conventional Banks-Casher relation, however, we shall refer to the above as the spectral density. The integration contour should go around all of the poles at $\lambda_{i}$ to pick all the eigenvalues up. In our notation $\langle\langle\cdots\rangle\rangle$ represents the ensemble average including the Dirac determinant.

Here we consider the contour which is an infinitely large circle in the complex plane surrounding all the poles. Then the contour integral must amount to zero because $\rho_{\chi}(\lambda) / \lambda$ goes to zero faster than $|\lambda|^{-1}$. That means that we can evaluate the above integral by the negative residue of the pole at $\lambda=0$. After all, we have

$$
\langle\bar{\psi} \psi\rangle=-N_{f} \pi\left\langle\left\langle\rho_{\chi}(0)\right\rangle\right\rangle .
$$




\subsection{Diquark Condensate}

We can develop the same argument for the diquark condensate as well as the chiral condensate. We shall limit our discussions to the case with degenerate two-flavor $(u$ and $d$ ) quarks. In the presence of the same quark chemical potential $\mu$ for $u$ and $d$ quarks, we can introduce into the Lagrangian density the source for the diquark $J$ and for the anti-diquark $\bar{J}$ which are anti-symmetric in spin, color, and flavor. By means of a variable change by $\bar{\phi}_{d} \equiv \psi_{d}^{T} C \sigma_{2}$ and $\phi_{d} \equiv C \sigma_{2} \bar{\psi}_{d}^{T}$, it is possible to write the Lagrangian density down as

$$
\mathscr{L}=\left(\bar{\psi}_{u}, \bar{\phi}_{d}\right)\left(\begin{array}{cc}
\mathscr{D}(\mu) & -J \gamma_{5} \\
\bar{J} \gamma_{5} & \mathscr{D}(-\mu)
\end{array}\right)\left(\begin{array}{l}
\psi_{u} \\
\phi_{d}
\end{array}\right) .
$$

The integration over the quark fields is then straightforward and the resultant partition function is given as the determinant as follows;

$$
Z(J)=\left\langle\operatorname{det}\left(\begin{array}{cc}
\mathscr{D}(\mu) \gamma_{5} & -J \\
\bar{J} & \mathscr{D}(-\mu) \gamma_{5}
\end{array}\right)\right\rangle_{U}=\left\langle\operatorname{det}\left[\mathscr{D}(\mu) \mathscr{D}^{\dagger}(\mu)+|J|^{2}\right]\right\rangle_{U},
$$

where we have used $\gamma_{5} \mathscr{D}(-\mu) \gamma_{5}=\mathscr{D}^{\dagger}(\mu)$. We note that $\mathscr{D}(\mu) \mathscr{D}^{\dagger}(\mu)$ is always Hermitean though $\mathscr{D}(\mu)$ may not be so. We can then prove that the eigenvalue of $\mathscr{D}(\mu) \mathscr{D}^{\dagger}(\mu)$ is non-negative real, which we denote by $\xi_{i}^{2}$ with choosing $\xi_{i} \geq 0$. The diquark condensate thus reads

$$
\left\langle\bar{\psi}_{u}\left(C \gamma_{5}\right) \sigma_{2} \bar{\psi}_{d}^{T}\right\rangle=\left.\frac{\partial}{V \partial J} Z(J)\right|_{J=0}=\frac{1}{V}\left\langle\left\langle\sum_{i} \frac{J}{\xi_{i}^{2}+|J|^{2}}\right\rangle\right\rangle=\pi\left\langle\left\langle\rho_{D}(0)\right\rangle\right\rangle,
$$

where we have defined the diquark spectral density,

$$
\rho_{D}(\xi)=\frac{1}{V} \sum_{i} \delta\left(\xi-\xi_{i}\right)
$$

\section{Eigenvalue Distribution for a Random Configuration}

In this article we will take only one random configuration as a representative instead of calculating the ensemble average over many random configurations. Actually the eigenvalue distribution for one typical gauge configuration turns out to be quite informative in our case. This simplification is legitimate because each random configuration equally contributes to a physical quantity in the strong coupling limit.

The Dirac operator at finite density in the staggered fermion formalism is

$$
\begin{array}{r}
\mathscr{D}(\mu) \equiv m_{q} \delta_{m, n}+\frac{1}{2} \sum_{i} \eta_{i}(m)\left[U_{i}(m) \delta_{m+\hat{\imath}, n}-U_{i}^{\dagger}(n) \delta_{m, n+\hat{\imath}}\right] \\
+\eta_{4}(m)\left[\mathrm{e}^{\mu} U_{4}(m) \delta_{m+\hat{4}, n}-\mathrm{e}^{-\mu} U_{4}^{\dagger}(n) \delta_{m, n+\hat{4}}\right]
\end{array}
$$

where $\eta_{\mu}(n) \equiv(-1)^{n_{1}+n_{2}+\cdots+n_{\mu-1}}$.

The zero-density Dirac operator in the staggered fermion formalism is anti-Hermitean except for the mass term, so that all the eigenvalues reside on a line whose real part is $m_{q}$. The chemical potential breaks anti-Hermiticity and the eigenvalue distribution has a width along the real axis as 
$\mu$ goes larger. The broadened width in the real direction has a definite physical meaning. In the case of $m_{q} \neq 0$ the distribution has to be shifted by $m_{q}$ and then the entire eigenvalue distribution can be placed in the positive quadrant as long as $\mu$ is small as compared to $m_{q}$. It is hence a natural anticipation that the superfluidity has an onset when the eigenvalue distribution becomes as wide as it reaches the origin.
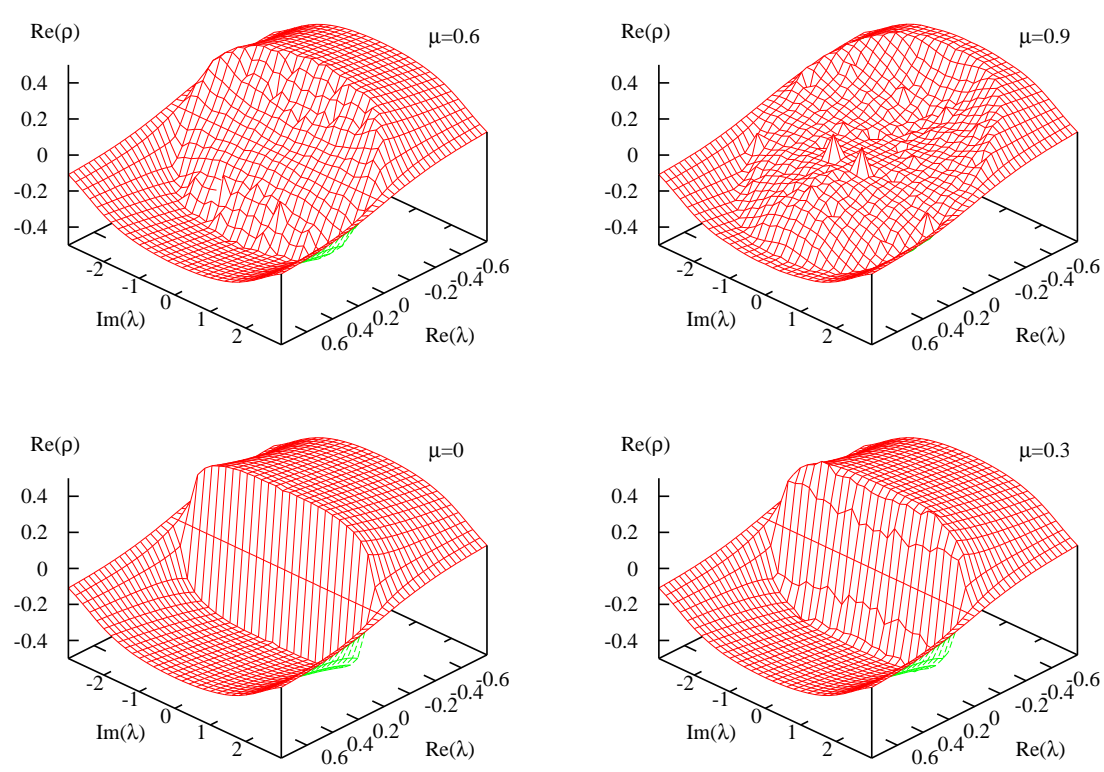

Figure 1: Real part of the resolvent, $\operatorname{Re}\left(\rho_{\chi}(\lambda)\right)$, in the complex plane for various values of the chemical potential.

We can explicitly calculate all the eigenvalues and the spectral density (3.2) to evaluate the chiral condensate through the Banks-Casher relation in Eq. (3.3). Because of the quartet pattern of the eigenvalue distribution the imaginary part of $\rho_{\chi}(\lambda)$ is vanishing on the real axis. We show the real part of the spectral density (resolvent) (3.2) in Fig. 1 for various $\mu$. It is remarkable that the spectral density for a random configuration looks such smooth even without taking an ensemble average. As we have mentioned, the eigenvalues and thus the spectral density with a finite $m_{q}$ can be deduced simply by a shift along the real axis by $m_{q}$. Therefore, $\rho_{\chi}(0)$ appearing in Eq. (3.3) can be read from Fig. 1 by the value at $(\operatorname{Re} \lambda, \operatorname{Im} \lambda)=\left(-m_{q}, 0\right)$.

When $\mu=0$ a sharp perpendicular wall stands at $\operatorname{Re}(\lambda)=0$ which is responsible for a nonvanishing chiral condensate in the limit of $m_{q} \rightarrow 0$ while keeping $\mu=0$. The wall is smoothened by the effect of $\mu \neq 0$ and it is no longer vertically upright at finite density, which leads to an interesting observation. In fact, it is not hard to conceive from Fig. 1 that the chiral condensate becomes zero in the chiral limit while keeping infinitesimal but nonzero $\mu$. This is absolutely consistent with Ref. [1].

We shall next evaluate the diquark condensate using the Banks-Casher relation (3.6). We will start with the chiral limit $\left(m_{q}=0\right)$ and then go into the finite mass case that we choose $m_{q}=0.2$ 
here. For convenience we define the integrated diquark spectral number,

$$
n_{D}(\xi)=\int_{0}^{\xi} \mathrm{d} \xi^{\prime} \rho_{D}\left(\xi^{\prime}\right)
$$

whose slope at $\xi=0$ gives the spectral density $\rho_{D}(\xi=0)$ which is proportional to the diquark condensate. Although the staggered fermion Lagrangian does not involve the Dirac spinor, it is not difficult to make use of the Nambu-Gor'kov representation to express the diquark condensate by the diquark spectral density. Since the derivation is only straightforward, we will not reiterate it but skip detailed arithmetics. To summarize the resultant relations, we can prove that

$$
\sigma \equiv \frac{1}{2}\langle\bar{\chi} \chi\rangle=\frac{\pi}{2} \rho(0), \quad \Delta \equiv \frac{1}{2}\left\langle\chi \mathrm{i} \sigma_{2} \chi\right\rangle=\frac{\pi}{4} \rho_{D}(0),
$$

where the extra $1 / 2$ factor in the diquark relation comes from the square-root prescription necessary to cancel the doubled Nambu-Gor'kov basis. In the above we have chosen the same normalization as Ref. [4].
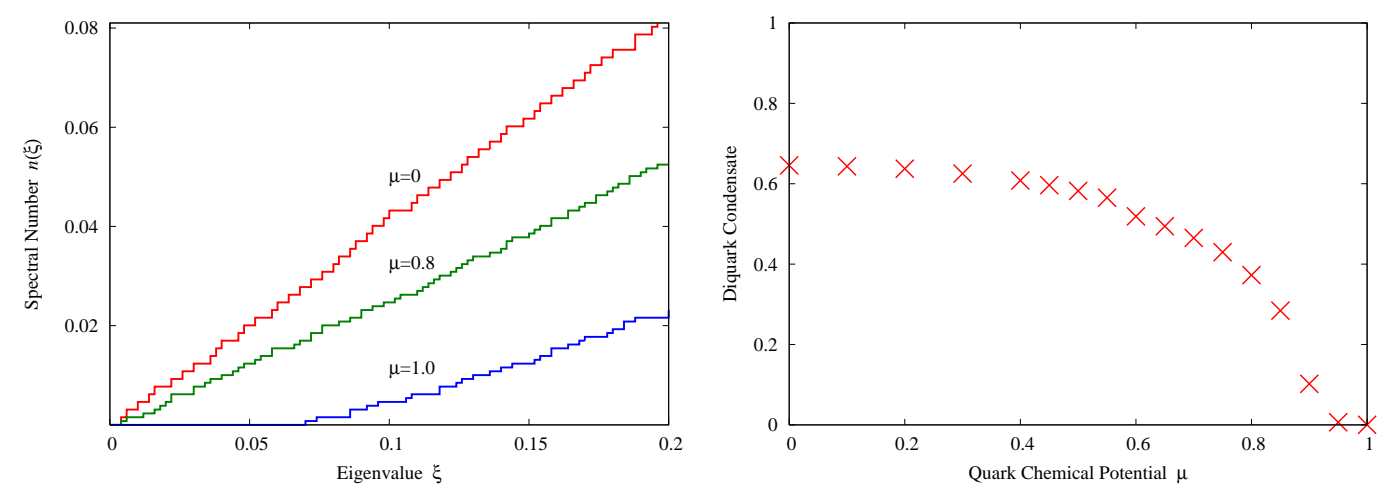

Figure 2: Left) Histogram of $n_{D}(\xi)$ whose slope gives the spectral density $\rho_{D}(\xi)$. Right) Diquark condensate as a function of $\mu$ at $m_{q}=0$.

It is intriguing to evaluate $n_{D}(\xi)$ by the explicit numerical calculation for the eigenvalues in Fig. 1 from which we can get $\rho_{D}(\xi)$. Figure 2 shows our results in the chiral limit. In this case only the diquark condensate is a non-vanishing quantity [1]. We plot the diquark condensate in the right of Fig. 2 without indicating the error bar. We did so because, though the fitting error is small, the systematic error is large. If we change the working procedure to measure the slope from the histogram in the left of Fig. 2, the resultant diquark condensate would change too. For clarity of our numerical procedure we explain how we compute the slope of $n_{D}(\xi)$ at the origin. We assume a functional form $n_{D}(\xi)=a \xi+b \xi^{2}$ within the range $\xi \in[0,0.1]$ and fix $a$ and $b$ to fit the data. Then, $a$ gives the slope at the origin. If $a$ turns negative, that means no spectral density at the origin, and so the diquark condensate should be zero. In this way we draw the right of Fig. 2 which shows outstanding agreement with the upper-left of Fig. 1 in Ref. [4].

The $m_{q}$ dependence in $\mathscr{D}(\mu) \mathscr{D}^{\dagger}(\mu)$ is not such trivial as in the case of $\mathscr{D}(\mu)$. Roughly speaking, a finite $m_{q}$ shifts the eigenvalue in the positive real direction so that the eigenvalue distribution is blocked in the vicinity of the origin as long as $\mu$ is small. For $\mu$ above a certain threshold value the diquark spectral density becomes finite at $\xi=0$, and the diquark condensation is activated. 
We can repeat the calculation in the massive case as well. Our final results are presented below in Fig. 3. We note that the onset for the chiral condensate decrease is determined by the front edge of the sidling wall which corresponds to the edge of the Dirac eigenvalue distribution, which in turn corresponds to the diquark onset.
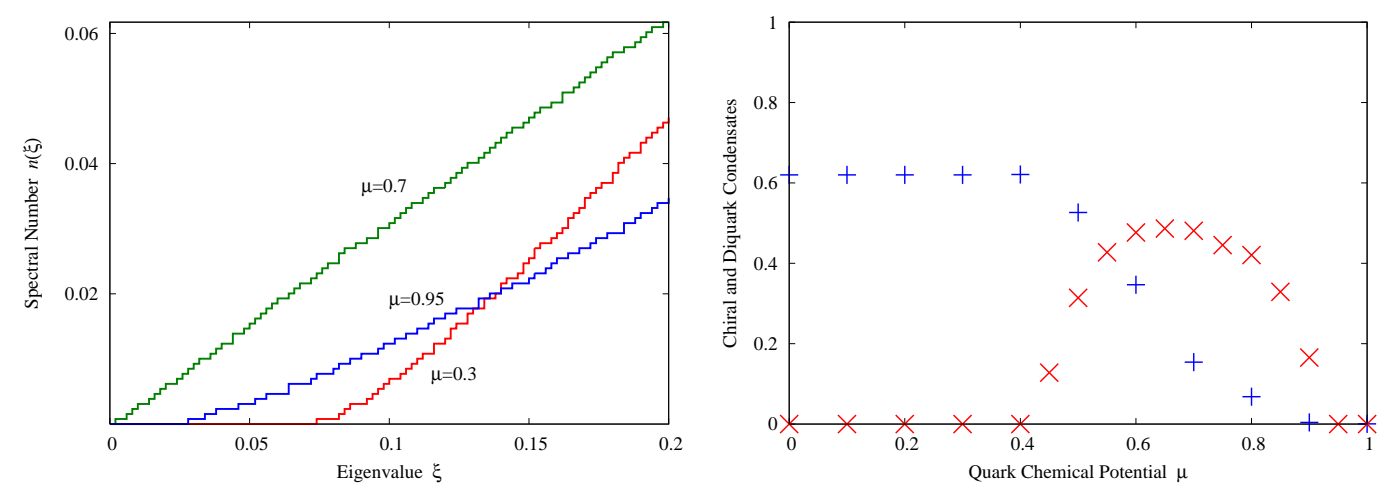

Figure 3: Left) Histogram of $n_{D}(\xi)$ in the case of $m_{q}=0.2$. Right) Chiral and diquark condensates as a function of $\mu$ at $m_{q}=0.2$.

It is impressing that the results in the right of Fig. 3 is consistent qualitatively with the meanfield analysis in the strong coupling limit given in the upper-left of Fig. 1 in Ref. [4], though the direct comparison is not possible for different mass choice.

The technique developed here can be applied to the research on the QCD phase diagram [13] including the diquark condensate $[8]$ in the future.

\section{References}

[1] E. Dagotto, F. Karsch and A. Moreo, Phys. Lett. B 169, 421 (1986).

[2] R. Rapp, T. Schafer, E. V. Shuryak and M. Velkovsky, Phys. Rev. Lett. 81, 53 (1998).

[3] S. Hands, I. Montvay, S. Morrison, M. Oevers, L. Scorzato and J. Skullerud, Eur. Phys. J. C 17, 285 (2000).

[4] Y. Nishida, K. Fukushima and T. Hatsuda, Phys. Rept. 398, 281 (2004).

[5] G. Akemann and E. Bittner, Phys. Rev. Lett. 96, 222022 (2006).

[6] K. Fukushima, JHEP 0807, 083 (2008).

[7] K. Fukushima, PoS LATTICE2007, 185 (2007).

[8] V. Azcoiti, G. Di Carlo, A. Galante and V. Laliena, JHEP 0309, 014 (2003).

[9] For a recent review; see, M. G. Alford, A. Schmitt, K. Rajagopal and T. Schafer, arXiv:0709.4635 [hep-ph].

[10] T. Banks and A. Casher, Nucl. Phys. B 169, 103 (1980).

[11] H. Kluberg-Stern, A. Morel and B. Petersson, Nucl. Phys. B 215, 527 (1983).

[12] K. Fukushima, Prog. Theor. Phys. Suppl. 153, 204 (2004).

[13] N. Kawamoto, K. Miura, A. Ohnishi and T. Ohnuma, Phys. Rev. D 75, 014502 (2007). 